\title{
La importancia estratégica del Ártico en la geopolítica marítima
}

\author{
Silvia Marina Rivas \\ de Hernández
}

Recibido: 05/03/2021

\author{
silvia.rivas@ues.edu.sv \\ Universidad de El Salvador (El Salvador)
}

Relaciones Internacionales

Resumen: Como consecuencia del deshielo polar sufrido en los últimos años por el calentamiento global, la ruta marítima ártica cobró importancia frente a las rutas tradicionales que comprenden el paso por los estrechos de Malaca y Suez. Según estudios de Nature Climate Change (2020), se espera que para 2035, la ruta del Norte pueda ser viable para el transporte de mercancías como resultado del incremento de las temperaturas y desaparición de varias capas de hielo polar. En este artículo se pretende describir la relevancia geopolítica de la región ártica para los Estados ribereños: Canadá, Dinamarca, Estados Unidos, Noruega y Rusia, así como sus pretensiones en los próximos años, no solo para los 5 del Ártico sino para otras potencias como China, que sin ser un país ribereño, tiene objetivos claros sobre la zona. A través de una investigación documental sobre las prioridades de estos países plasmadas en los programas estratégicos sobre la región y utilizando la teoría del realismo estructural como marco de análisis, se relata cómo los países ribereños del Polo Norte han establecido acciones desde diferentes ámbitos para el logro de sus objetivos, atendiendo a sus capacidades dentro de la estructura internacional.

Palabras clave: geopolítica, Ártico, rutas marítimas, cambio climático, realismo estructural, seguridad marítima, estructura internacional

* Cómo citar: Rivas de Hernández, S. M. (2021). La importancia estratégica del Ártico en la geopolítica marítima. Relaciones Internacionales, 30 (61), 140.

https://doi.org/10.24215/23142766e140

Editor: Juan Alberto Rial, Instituto de Relaciones Internacionales Facultad de Ciencias Jurídicas y Sociales (Universidad Nacional de La Plata)
Entidad editora: Relaciones

Internacionales, es una publicación del Instituto de Relaciones Internacionales (Facultad de Ciencias Jurídicas y Sociales (Universidad Nacional de La Plata Argentina)

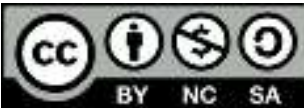

Reconocimiento-NoComercial Compartirlgual 4.0 Internacional (CC BY-NC-SA 4.0) 


\title{
The strategic importance of the Arctic in maritime geopolitics
}

\section{Silvia Marina Rivas de Hernández ${ }^{1}$}

\begin{abstract}
As a consequence of the polar melting suffered in recent years due to global warming, the Arctic sea route has gained importance over the more traditional routes, including the passage through the Straits of Malacca and Suez. According to the studies carried out by Nature Climate Change (2020), by 2035 it is expected that the so-called Northern route may be fully available for the transport of goods, due to the increase in temperatures and the disappearance of important polar ice caps. This article aims to describe the geopolitical relevance of the Arctic region for the coastal states: Canada, Denmark, the United States, Norway and Russia, as well as the claims over it in the coming years not only by the the Arctic's Five but also by such other powers as China, which - while it is not a coastal country- has clear objectives regarding the area. Through documentary research on the priorities of these States, the strategic programs on the region and using the theory of structural realism as a framework of analysis, it is reported how the coastal countries of the North Pole have established actions from different spheres to achieve their objectives based on their capabilities within the international structure.
\end{abstract}

Key words: geopolitic, Artic, maritime routes, global warming, structural realism, maritime security, international estructure.

\section{Introducción. Aspectos generales}

El consumo masivo y la producción en masa desde la época industrial derivó en daños al ecosistema, lo que incluye la emisión de gases de efecto invernadero, cuya consecuencia ha sido el aumento de la temperatura del planeta y, por ende, la existencia del cambio climático ${ }^{2}$. Al experimentar temperaturas más cálidas, los casquetes polares comienzan a derretirse a un ritmo mayor; así, lo que estaba cubierto por hielo denso, hoy se encuentra libre

1 Licenciada en Relaciones Internacionales de la Universidad de El Salvador. Máster en Seguridad, Paz y Conflictos Internacionales de la Universidad Santiago de Compostela en Galicia, España. Docente de la Escuela de Relaciones Internacionales de la Facultad de Jurisprudencia y Ciencias Sociales de la Universidad de El Salvador.

2 Según la Convención Marco de las Naciones Unidas sobre el Cambio Climático en su artículo1, numeral 2 cambio climático se entiende un cambio de clima atribuido directa o indirectamente a la actividad humana que altera la composición de la atmósfera mundial y que se suma a la variabilidad natural del clima observada durante períodos de tiempo comparables. Organización de las Naciones Unidas, Convención Marco de 
o con una ligera capa de este que no representa peligro para los buques rompehielos diseñados para las travesías en mares congelados.

La extensión de hielo marino en el Polo Norte experimentó descensos en los últimos años; por ejemplo, el mínimo histórico que se registró en 2012 (National Snow and Ice Data Center, s. f.) dejó buena parte de los mares árticos despejados de hielo, situación que resulta benéfica no solo para el paso de barcos, sino también para la extracción de minerales y petróleo ${ }^{3}$. Los países ribereños muestran interés por delimitar sus sectores de soberanía y jurisdicción, con ideas de aprovechar estos recursos y la utilización de las rutas marítimas del norte, no solo en términos económicos sino en cuanto al posicionamiento político global dentro de la estructura de poder internacional.

El objetivo de este artículo es describir la relevancia de la zona ártica para los países ribereños a fin de comprender sus estrategias de posicionamiento según lo reflejado en sus programas estratégicos de acción sobre la región. Si bien este tema no es nuevo como problema de investigación, constituye un campo de estudio importante para las Relaciones Internacionales a partir de la configuración de esfuerzos globales y regionales por la conservación ambiental frente al cambio climático dentro de un sistema de transición en donde potencias emergentes, como Rusia y China, pretenden consolidar un mundo multipolar frente al unipolarismo después de la finalización de la guerra fría. Esto se explicará más adelante al utilizar el realismo estructural como marco de análisis. Así, se afirma que, en la medida que los Estados ribereños y China modifiquen la estructura de poder en la zona ártica logrando sus aspiraciones de posicionamiento, la cooperación entre los 5 del Ártico podría verse ralentizada en materia de conservación de la región.

Este documento aborda diferentes aspectos desde una lógica deductiva que ayudan a comprender la importancia del Ártico como zona geopolítica y estratégica. En ese sentido, se abordan cuestiones generales que explican los alcances, limitaciones y abordaje teórico del tema. Seguidamente, se describen las características del Ártico como espacio geográfico y estratégico, y acotadamente se incluye información del impacto del cambio climático en la zona polar ártica. El tercer apartado expone cada una de las posiciones de los Estados involucrados a fin de comprender sus prioridades y acciones en la zona. Las rutas marítimas del norte son abordadas en un cuarto apartado para ilustrar la posibilidad de uso como alternas a las rutas de Suez y Panamá. Finalmente, se elaboran algunas prospectivas sobre el tema y conclusiones del posible comportamiento de los Estados ribereños en los próximos años.

\subsection{Metodología}

Para el desarrollo de este artículo de naturaleza descriptiva y bibliográfica se utilizaron

las Naciones Unidas sobre el Cambio Climático, 9 de mayo de 1992, https://unfccc.int/resource/docs/convkp/convsp.pdf, fecha de consulta: 28 de septiembre de 2020.

3 Se considera que el $30 \%$ de las reservas de mundiales de gas natural y el 13\% de las de petróleo se encuentran en esta región. Palacián de Inza, Blanca, La Creciente Importancia del Ártico, Análisis, Instituto Español de Estudios Estratégicos, octubre 2010, pág. 50 
fuentes primarias como la revisión de los programas estratégicos estatales sobre la zona ártica y las publicaciones del Consejo Ártico, también se consultaron fuentes secundarias entre las que se encuentren diferentes investigaciones sobre la temática ártica en distintas dimensiones. Los tipos de documentos fueron variados e incluyen informes, libros, artículos, documentos de análisis y opinión, revistas científicas, entre otros, tanto en idioma español como en inglés. Estas publicaciones fueron consultadas exclusivamente por medio de sitios web en atención a las medidas de bíoseguridad con respecto a la COVID19 que desde el 2020 afecta a la población mundial.

\subsection{Limitaciones temáticas}

Los Estados ribereños en estudio para este artículo son Canadá, Dinamarca, Estados Unidos, Noruega y Rusia, los cuales tienen sus propias pretensiones estratégicas en la región e incluso diferendos pendientes en la Corte Internacional de Justicia sobre la ampliación de su plataforma continental a través de la Dorsal Lomónosov, cuestión que se explicará más adelante. No se incluirán las prioridades y programas estratégicos de Finlandia, Islandia y Suecia, que, aunque son Estados vecinos y miembros del Consejo Ártico, no son Estados ribereños puesto que su salida al mar está situada dentro del Mar Báltico y el Mar de Barents (Zaragoza, 2014). La inclusión de China está justificada por las acciones exteriores que este país lleva a cabo en materia de inversión y lazos comerciales con países ribereños para asegurarse de obtener ventajas logísticas sobre las rutas marítimas del norte. Además, este país asiático es uno de los pioneros en inversión e investigación en el Ártico desde 1995 y se encuentra muy activo en las cuestiones sobre las capacidades políticas y legales en la región al considerar que las decisiones sobre el polo no pueden ser exclusivas de los Estados ribereños (Heske, 2015).

\subsection{Estado del arte}

En cuanto a la temática del Ártico existe diversa bibliografía que nutren las visiones sobre la situación de este mar congelado. Entre las investigaciones que se han llevado a cabo se pueden encontrar visiones geopolíticas, medio ambientales, marítimas y de seguridad, no solo para los países ribereños sino para otros Estados vecinos entre los que figuran europeos y asiáticos.

En términos geopolíticos, varios autores explican la importancia estratégica del ártico basados en la presencia de recursos energéticos y pesqueros en la zona. Se aportan datos las rutas árticas del norte y se retoman los programas estratégicos de los diferentes Estados miembros del Consejo Ártico a fin de desvelar sus intenciones en la región según el comportamiento mostrado a través de la Política Exterior de cada uno de estos. Otras investigaciones consultadas se relacionan con las rutas árticas o rutas del norte y el interés de los Estados ribereños por lograr el control y dominio de estas. También se recopilaron algunos documentos relacionados con la situación ambiental del Ártico y su deshielo para lograr una visión más amplia sobre las implicaciones de los programas impulsados por los Estados interesados en la zona. Finalmente hay documentos que incluyen los instrumentos legales aplicables al ártico y su conservación.

A pesar de que las investigaciones sobre el Ártico son variadas y vastas, una pequeña 
muestra aborda el tema desde la disciplina de las Relaciones Internacionales. La importancia para su estudio parte de las implicaciones dentro de la estructura de poder en la que se ubican los países ribereños y las estrategias para la consecución de sus objetivos. En consecuencia, el logro de estos podría modificar el actual sistema internacional otorgando ventajas y un mejor posicionamiento global a quien predomine en la zona, lo que afectaría las relaciones entre Estados. En ese sentido este artículo, a diferencia de lo desarrollado por otros autores en los que se apoya este documento, plantea las acciones de los países ribereños dentro de una visión de realismo estructural y describe a grandes rasgos la importancia estratégica del Ártico y las implicaciones de una posible modificación de la estructura de poder en la región.

\subsection{Marco teórico}

Para explicar la posición de los Estados y la importancia geopolítica del las aguas y la plataforma ártica, se aplicará el Realismo Estructural como teoría. Según el realismo estructural propuesto por Keneth Waltz, la finalidad de los Estados se encuentra en la capacidad de garantizar su seguridad y no en lo que se planteó dentro del realismo clásico de Morgenthau, en el cual el poder constituye el fin del Estado (Waltz, 1959). Waltz propone que, dentro de la relaciones estatales, existe una especie de estructura tácita en la que aquellos países con mayor poder, traducido en capacidad militar y de influencia, se encuentran en la cúspide de esa estructura, de forma que gozan también de la capacidad de maniobra dentro de esta y, por ende, de mayor seguridad (Sanahuja \& Del Arenal, 2015).

El realismo estructural considera las capacidades de los Estados para mantenerse en cierta posición dentro de la estructura internacional, por lo que aquellos con fuerte desarrollo militar, político, económico y diplomático se colocarán en mejores posiciones que aquellos que no cuentan con capacidades igualables. Los Estados con menores capacidades serán, por lo tanto, más vulnerables ante cualquier amenaza, por lo que es importante establecer alianzas estratégicas, acuerdos y entendimientos con otros para neutralizar las posibilidades de conflicto en un sistema anárquico (Hernández, 2008) .

En esta estructura es probable que alguno de los países que se encuentran en la parte intermedia considere la posibilidad de ascender; para ello deberá hacer un análisis de sus factores sistémicos ${ }^{4}$, capacidades militares, políticas, económicas e inclusive diplomáticas, a sabiendas de que las maniobras que lleve a cabo deberán de ser lo suficientes como para generar una ruptura, puesto que las potencias que se encuentran en la cumbre harán todo lo posible por mantener el statu quo. En este sentido, el dilema de seguridad ${ }^{5}$ latente para

\footnotetext{
4 En este punto retomando lo propuesto por Pearson y Rochester en el cual los factores sistémicos de un Estados se clasifican como aquellas condiciones externas que no pueden ser controlados, entre ellos se encuentran: la geografía, los países vecinos, y, para algunos países que se encuentran en la llanura de la estructura de poder el Sistema Internacional en el que se insertan. Pearson, Frederick y Rochester, Martin, Situación Global en el Siglo XXI, Editorial Mc Graw Hill, $4^{\circ}$ Edición, Colombia, 2000
}

5 El dilema de seguridad es una premisa propuesta por John Herz, en la que los Estados al estar sometidos a 
los Estados ribereños establecido en la zona a través de las pretensiones asertivas de Rusia sobre el dominio de sus costas con el despliegue de armamentos y bases militares ${ }^{6}$ (Gómez, 2019) genera que países con tradiciones de comportamiento exterior más aislacionista y concertadoras, como Noruega, se muestren proactivos sobre los temas del Ártico dentro de espacios de defensa multilaterales como la alianza del Atlántico Norte.

Dadas las características de Noruega, por su menor capacidad militar que Rusia, decide cooperar con los rusos por una parte, pero advertir por la otra en el seno de la OTAN de no establecer acciones directas. Mientras las tensiones se han mantenido controladas en la zona aun con las demostraciones de expansión por parte de Rusia y el cese de cooperación entre esta y Noruega, la estructura de poder actual junto con la expansión de la OTAN hacia el este a partir de la posguerra fría exacerbaron la percepción de Rusia sobre su seguridad, por lo que se ha comportado de forma ofensiva para reinvidicar su ventaja en el Ártico, cuestión que será abordada más adelante.

\subsection{Marco conceptual}

El desarrollo de este artículo versa sobre tres conceptos elementales: geopolítica, seguridad marítima y estructura internacional de poder. La relación entre estos conceptos con sus referentes empíricos explicada a los largo del documento regirá el análisis del comportamiento de los Estados ribereños en la búsqueda de sus objetivos árticos.

\subsubsection{Geopolítica:}

El término Geopolítica fue acuñado por el politólogo Rudolf Kjellén como una extensión del poder y jurisdicción del Estado sobre el espacio geográfico. La geopolítica se define como la influencia de los factores geográficos, en las más amplia acepción de la palabra, en el desarrollo político de los pueblos y Estados (Cuellar Laureano, 2012). Así, las características geofísicas, la posición, el clima y los recursos del subsuelo existentes dentro del Estado influyen en la política y el comportamiento de este en la consecución de sus objetivos. En otra acepción del término, Karl Haushoffer, citado por Londoño (2018) , amplía el concepto de geopolítica como la conciencia geográfica del Estado. Ella proporciona la materia prima de donde el hombre de Estado, de espíritu creador, obtiene su obra de arte.

La geopolítica como estudio incluye la relación entre los ámbitos geográficos: tierra, mar y aire con el ejercicio del poder de los Estados en estos espacios. El aprovechamiento de las características geográficas del país en favor de los objetivos trazados constituyen el reto de cualquier hombre/mujer de Estado. En el caso del Ártico la importancia geopolítica

una anarquía internacional buscarán establecer las estrategias necesarias para sentirse más seguros esforzándose por alcanzar esa seguridad a través de adquirir más poder e influencia, los que no tengan esa capacidad buscarán aliarse con otros para hacer frente a posibles amenazas de otros Estados más beligerantes, reproduciendo un círculo vicioso de seguridad y acumulación de poder. Herz, J. El internacionalismo idealista y el dilema de la seguridad. Política mundial, 2 edición, 1950 pp. 157-180.

6 Aunque el dilema de seguridad forma parte de los desarrollos posteriores a Waltz no será abordado en este artículo. 
de la región está ligada a las características propias del Polo Norte y la capacidad de dominio y ejercicio del poder de cada Estado frente a sus vecinos a fin de no quedar vulnerables ni relegados en esa relación. Así, cada país traza estrategias ajustadas a sus capacidades políticas y militares en la búsqueda de sostener un equilibrio de poder que no necesariamente tiene que ver con equidad. Para este artículo se entenderá como geopolítica la acepción de Laureano en la que se reconoce la influencia de los factores geográficos en el desarrollo político de los Estados, siendo la política la variable dependiente en la relación entre ambas.

\subsubsection{Seguridad Marítima}

La Seguridad Marítima se define como todo lo relativo a la protección de vidas y bienes a través de las normativas, la gestión y la tecnología de todas las formas de transporte por agua, no solo a través del mar (Larrucea, 2015, p. 13) , lo que implica el desarrollo de leyes en distintos niveles y dimensiones para garantizar la protección de ambos mientras son transportados por medios marítmos.

El Departamento de Seguridad Nacional español amplía esta definición al integrar algunos rasgos establecidos por la Estrategia de Seguridad Marítima de la UE en la que se indica que la seguridad marítima debe contar con la garantía del respeto y aplicabilidad del Derecho Internacional, leyes nacionales y la libre navegación, así como la protección integral de las personas, infraestructura, medio ambiente, recursos y transporte (Deparamento de Seguridad Nacional, s. f.) . La Seguridad Marítima engloba, en este orden de ideas, normativas y procesos que actúan tanto en sentido preventivo como en respuesta a situaciones emergentes, entre las que se encuentran en las regulaciones de la $\mathrm{OMI}^{7}$ respecto a operaciones de rescate y salvamento de naúfragos, así como la lucha contra la contaminación marítima (Suárez Llanos-Galán, 2009) .

Los Estados deben ser responsables de generar los marcos legales necesarios para la protección de la vida y los recursos en el mar, más allá de la simple normativa para el transporte marítimo. Implica no solo la aplicación del Derecho Internacional, los tratados y acuerdos establecidos como Estados parte en la zona, sino también el cumplimiento de los compromisos asumidos para la conservación del ambiente y las medidas globales para detener la contaminación marítima y la depredación de las especies.

La garantía de la aplicación de la normativa y la protección del medio ambiente en el Ártico no necesariamente irá en desmedro de los intereses geopolíticos de los países ribereños, pero su existencia depende de los niveles de cooperación entre los Estados a escala global para frenar los efectos del cambio climático y el deshielo polar. Con la posibilidad de la utilización de las rutas marítimas del norte por el deshielo como alternativa a las tradicionales, los Estados circumpolares deberán lograr mayores compromisos en la protección de comunidades indígenas, fauna y flora marina, así como protocolos de prevención y respuesta ante una posible contaminación de las aguas por el paso de los buques. 


\subsubsection{Estructura internacional}

Tal como se abordó en el marco conceptual, el realismo estructural plantea la existencia de una estructura tácita dentro de la anarquía como característica sine qua non del sistema internacional. Los Estados constituyen la unidad principal de análisis de la teoría de Waltz, las interacciones regulares entre los Estados de distinta índole dotan de una especie de organización al caos en el que estos se mueven. Las características de la estructura dependen de la naturaleza de tales interacciones entre estos y la posición que ostentan según sus capacidades y cómo se expresan en la arena internacional (Hernández, 2008, p. 18) .

Dentro de los actores involucrados en el Ártico se encuentran Estados potencias de acuerdo a sus capacidades: Estados Unidos, Rusia y China; los dos últimos, considerados como países emergentes. Ante una estructura internacional que privilegia a Estados Unidos como hegemón después de la guerra fría, bajo una asociación flexible y multilateralista (Brzezinski, 1998) , tanto China como Rusia desplazan sus esfuerzos en la búsqueda de una mejor posición dentro de la estructura internacional que les consolide como potencias globales en un mundo multipolar. En consonancia, las acciones implementadas de cara a la obtención de ventajas en espacios estratégicos como el Polo Norte estarán marcadas por sus metas en la región. Dinamarca, Canadá y Noruega, como potencias occidentales medias (Valencia \& Ruvalcaba, 2010), al no poseer una capacidad económica y militar comparable con los países mencionados anteriormente, tendrán que crear sus propias estrategias sin descartar el establecimiento de acuerdos en espacios multilaterales como el Consejo del Ártico, las Naciones Unidas, entre otros, para no perder sus posiciones actuales y ser relegadas en sus propias intenciones.

\section{Descripción geográfica y estratégica del Ártico}

Siendo uno de los espacios geográficos del planeta con mayor extensión y recursos naturales, la zona polar ártica se diferencia del polo sur por estar constituida por masas de agua congelada y tener poca profundidad, en comparación con los demás océanos que bañan la superficie terrestre.

\subsection{Carácterísticas geográficas}

El océano ártico es el espacio geográfico más al norte del planeta, ubicado en el Polo Norte, que equivale al 2, $8 \%$ de la superficie terrestre y al 3, $9 \%$ de los océanos. Es el menor en profundidad, con 1, $05 \mathrm{~km}$ de hondura (Geoenciclopedia, s. f.). Durante el invierno una buena parte de sus aguas se convierten en hielo espeso, mientras que en el verano el derretimiento polar reduce una cantidad de hielo flotante y permite la navegación. Este deshielo implica una reducción del $12 \%$ cada década; sin embargo, se alcanzó un pico histórico en 2012, cn la reducción de la superficie congelada en 4 millones de kilómetros, de los 14 millones de extensión total, características que le hacen el más pequeño de las masas de agua del planeta (Lojo Romero, 2018). Se considera que en el Ártico y su región adyacente habitan cerca de 4 millones de personas nacionales de los Estados ribereños y Estados vecinos (Greenpeace, 2014) . A su vez es el hogar de mamíferos y aves migratorias, varios de estos en peligro de extinción (Sánchez, 2021). 
Al delimitar geográficamente la región ártica se utilizan diferentes referencias. Entre estas se encuentran los parámetros basados en datos isotérmicos, en los que el clima no sobrepasa los $10^{\circ} \mathrm{C}$ durante el verano. Otra variable de medición más exacta hace referencia a la ubicación de los territorios árticos en la líneas de los 66 33’ de latitud norte, que incluye ocho países colindantes: Canadá, Dinamarca, Estados Unidos, Finlandia, Islandia, Noruega, Rusia y Suecia (Gómez, 2019, p. 12).

Para este artículo, como se definió en el apartado de metodología, se tomarán los países que conforman el alto Ártico (High Artic).

\subsection{Recursos y ventajas estratégicas}

Estudios que datan desde 1962 en la URSS y 1967 en Estados Unidos descubrieron cerca de 61 campos de petróleo y gas natural en cuatro de los cinco países ribereños: 42 se encuentran en Rusia, 6 en Alaska, 11 en Canadá y 1 en Noruega. Con el pasar del tiempo, agotadas algunas reservas de gas y petróleo, se reemplazaban por otras nuevas reservas probadas. En 2007 se consideró que las reservas rusas constituyen el 81\% de la totalidad de estas en los Estados árticos y el $25 \%$ de las reservas mundiales. Rusia y Estados Unidos son los que más petróleo producen, seguidos por Canadá y Noruega con una participación total del $28 \%$ en la extracción mundial de crudo (Jørgensen-Dahl, 2010). El USGS ${ }^{8}$ (2008) en su informe 3049 estimó que hay cerca de 90 mil millones de barriles de petróleo y 1, 669 billones de pies cúbicos de gas natural, y el $84 \%$ de estos posibles yacimientos se encuentran más allá de las costas árticas.

Las cifras de petróleo y gas estimadas por el USGS mencionadas anteriormente ilustran por sí mismas la riqueza y la importancia en términos económicos para los Estados ribereños, empero, los costos de inversión en infraestructura para la extracción de estos recursos implicaría costos aproximados de unos $\$ 26$ billones, al menos en 2007, sin contar con que la infraestructura ya existente para el suministro de petróleo, gas, carbón y energía eléctrica acorde a la demanda actual deberá ser reemplazada para 2030, asunto que elevaría los costos de producción a unos $\$ 120$ por barril por la cantidad de inversión requerida en el reemplazo de sistemas (Jørgensen-Dahl, 2010) .

La posición septentrional de este océano congelado ofrece la posibilidad de desarrollar una ruta marítima comercial privilegiada al acortar distancias entre el extremo oriental y occidental, con menos choke points ${ }^{9}$ que la ruta del canal de Suez, en donde se concentra el $90 \%$ del tráfico marítimo mundial (Estrada, 2020). Así los buques provenientes de China

8 Servicio Geológico de los Estados Unidos, USGS por sus siglas en inglés.

9 Un choke point ('punto de estrangulamiento') , también llamado cuello de botella es un lugar en el que una ruta se estrecha por razones geográficas, dando a ese punto un alto valor estratégico. Definición tomada de El Orden Mundial, ¿Qué es un choke point?, https://elordenmundial.com/que-es-un-chokepoint/\#: :text=Los\%20choke\%20points\%20son\%20puntos, punto\%20un\%20alto\%20valor\%20estrat\%C3\%A9gico., fecha de consulta 30 de septiembre de 2020 
hacia Europa y parte de América o viceversa no tendrían que detenerse en los puntos de tráfico como Malaca, Suez o Gibraltar.

La jurisdicción sobre la plataforma continental en algunos espacios de Ártico se encuentran en disputa a través de litigios territoriales en la Corte Internacional de Justicia, como los existentes entre Estados Unidos y Canadá; Dinamarca y Canadá; y Rusia, Canadá y Dinamarca, siendo este último conflicto el de intereses más ambiciosos puesto que cada uno persigue la ampliación de su plataforma continental a través de la posesión de la Dorsal Lomonósov ${ }^{10}$ para su derecho de explotación (Palacian de Inza, 2010) . Independientemente a quien pertenezca, el Estado poseedor tendría mayores ventajas sobre sus vecinos, teniendo en cuenta la ampliación de la plataforma continental y los derechos derivados de esta.

\subsection{Cambio climático y el deshielo ártico}

El cambio climático generado por el aumento de las temperaturas globales ha acelerado el derretimiento del Ártico, causando un deterioro en los ecosistemas y en la biodiversidad de la zona. Los efectos de este derretimiento incrementan el nivel de los océanos y crea cambios en los patrones de precipitación y sequía en el resto de países, por lo que los esfuerzos en materia de combate a los factores causantes de estos cambios deben ser asumidos por la totalidad del planeta.

En 1996, por acuerdo de los ocho países considerados ribereños del Ártico, se suscribe en Canadá la declaración de Ottawa para crear el Consejo del Ártico, el cual incluye las organizaciones de población indígena de la región (Iturralde, 2019). El objetivo del Consejo es la cooperación, coordinación e interación entre los países árticos, población indígena y otros habitantes. El tema principal en este foro es la protección y conservación del medio ambiente en el Ártico mediante líneas y grupos de trabajo definidos: acciones de adaptación, energía verde y resiliencia (Artic Council, s. f.). Actualmente trabaja sobre el Plan Estratégico Marino del Ártico 2015-2025 como marco de acción para la protección del ecosistema y promoción del desarrollo sostenible.

En enero de 2015 la capa de hielo en el Ártico fue la más baja en ese mes desde 1979. Las emisiones de gases y la combustión de petróleo genera cambios en las temperaturas que provocan el deshielo y despejan los espacios para la extracción de más hidrocarburos y el paso de buques. Entre menos hielo existe en los polos, menor será la capacidad de reflejar la radiación solar, por lo que la temperatura se eleva, estableciéndose así un círculo vicioso (Greenpeace, 2014).

Según Nick Golledge de la Universidad de Wellington, no existen precedentes de la aceleración del deshielo en el Ártico al menos en los últimos tres siglos. Diferente a lo que

10 Es una dorsal oceánica que se encuentra en el océano Glacial Ártico con una extensión de 1800 km, tanto Dinamarca como Rusia la reclaman como propia tratando de demostrar que esta pertenece a la plataforma de Groenlandia y Eurasia respectivamente. Afán por saber, Dorsal Lomonósov, publicado el 23 de enero de 2010, https://afanporsaber.com/tag/dorsal-lomonosov\#.XwfhICiuJEY, fecha de consulta 30 de septiembre de 2020 
comúnmente se cree, el deshielo en el ártico no es lineal sino que se acelera con los años. El deshielo sufrido por los polos desde comienzos del siglo XX ha incrementado el nivel del mar en un 30\% (Rodríguez, 2018). Así, el desafío en el Ártico se encuentra en la capacidad de los Estados para aunar esfuerzos a través del Consejo en pro de la conservación del medio ambiente a pesar de las existencia de una vasta riqueza en recursos energéticos.

\section{Estados ribereños, principales intereses y estrategias}

Aunque la cooperación entre los miembros del Consejo Ártico es importante, existen algunos litigios pendientes entre estos que han sido trasladados a la Corte Internacional de Justicia para ser dirimidos de acuerdo al Derecho Internacional. Por ejemplo, Dinamarca, Canadá, Estados Unidos y Rusia sostienen reclamaciones sobre el círculo polar ártico.

En este sentido, algunos Estados manejan sus propios argumentos para probar que las pretensiones de soberanía y extensión de la plataforma continental son legítimas, con lo que, al fallarse en beneficio de uno de estos otorgaría ventajas territoriales y marítimas sobre la zona. Los beneficios del deshielo en esta región y la posibilidad de la explotación de sus recursos no solo se enmarcan entre los países circumpolares, sino que incluyen a otros Estados no ribereños como China, que, aunque no es el único, es el que presenta mayor actividad. En este apartado se describen los intereses y estrategias de cada uno de los Estados ribereños y de China bajo la óptica del realismo estructural.

\section{Canadá}

En la zona ártica, Canadá tiene diferendos territoriales con países como Estados Unidos y Rusia. En el caso del conflicto con Estados Unidos, la disputa entre ambos países se centra en el paso del noroeste (Northwest Passage) rodeado por un archipiélago ártico entre ambos países, cuyo dominio y posesión daría ventajas en la ruta del Norte acortando los tiempos de navegación en la medida que las aguas congeladas cedan al aumento de la temperatura en la región (Godio, 2019). El argumento de los canadienses está basado en una teoría que data de inicios del siglo XX Ilamada Teoría de los Sectores ${ }^{11}$ (Iturralde, 2019) , aunque tal argumentación no es reconocida por el Derecho Internacional.

Atendiendo a sus capacidades militares en la zona frente a otros Estados ribereños, Canadá reforzó su posición a través de la adquisión de vehículos anfibios, mejoró las infraestructuras y encargó la construcción de seis buques patrulla (Estrada, 2020, pp. 18-19). A nivel estatal reforzó su presencia en los territorios que considera le corresponde; por ejem-

11 Fue una teoría pronunciada por el senador Pascual Poirier en abril de 1907, al declarar que las regiones en el polo norte no son más que la extensión de los países que lo rodean, de forma que, para determinar la soberanía de estos, basta con trazar líneas convergentes desde los territorios circumpolares. 
plo, en Canada's Arctic and Northern Policy Framework incluye elementos de desarrollo comunitario de la población inuit ${ }^{12}$. En tanto para asegurar el dominio de la zona como objetivo geopolítico, se establecieron metas de defensa del territorio ártico-canadiense. Canadá pretende colocarse como líder en la región mediante políticas innovadores de seguridad marítima, reconociendo los desafíos a los que se enfrentará la región en la encrucijada de intereses turísticos, comerciales y la conservación del ambiente (Government of Canada, 2019) .

A pesar de las pretensiones territoriales y la ampliación de la presencia militar, Canadá reconoce la necesidad de cooperación entre los Estados y los pueblos indígenas, coloca en su política ártica como prioridad la necesidad de crear normativas que mejoren su capacidad de respuesta ante emergencias de rescate y protección de turistas y nativos.

\section{Dinamarca}

A través de la provincia autónoma de Groenlandia, Dinamarca forma parte de los 5 del Ártico al poseer uno de los territorios ribereños más vastos en la región. Al igual que los demás Estados ribereños, tiene diferendos territoriales pendientes. Los daneses aseguran la pertenencia de la Dorsal Lomonósov a la placa continental americana y en consecuencia podrían extender su plataforma continental. A su vez, sostienen otro litigio con los canadienses por los límites en la Bahía de Baffin (Palacian de Inza, 2010) .

El comportamiento de Dinamarca dentro de la estructura internacional es más defensivo en comparación con otros países. A pesar de que existen diferencias limítrofes entre Canadá, Dinamarca y Rusia, hasta el momento no ha existido ruptura de relaciones o tensión en el área, al menos con los rusos, puesto que con Canadá son aliados OTAN, por lo que un desacuerdo mayor entre ambos no sería probable. El Estado danés, al igual que los demás países ribereños, estableción en 2011 un programa estratégico a mediano plazo para el provecho y posicionamiento en el Ártico a través de Kingdom of Denmark Strategy for the Arctic 2011-2020, el cual divide su accionar en cuatro campos de acción, entre los que se encuentran el desarrollo sostenible en el que incluye el bienestar social, protección y conservación del medio ambiente, políticas de explotación de recursos en la zona y un abierto compromiso para la garantía de la seguridad marítima a través de la cooperación con sus socios (Government of Kingdom of Denmark, 2011).

Dinamarca, consciente de la riqueza de sus territorios insulares y de las ventajas geopolíticas de la zona ártica, decidió a través del reino establecer un grupo de trabajo en las Islas Feroe y Groenlandia para la creación de normativas aplicables a las inversiones en estos espacios, a fin de cumplir con el compromiso de la conservación ambiental.

12 Es el nombre correcto con el que se designa a los pobladores del Ártico, dado que la palabra esquimal hoy se considera una denominación despectiva y se prefiere utilizar el término inuit, que significa, en la lengua inuktitut, personas o seres humanos. National Geographic, Los esquimales y su lucha diaria por la supervivencia, https://historia.nationalgeographic.com.es/a/esquimales-y-su-lucha-diaria-por-supervivencia_9790 


\subsection{Estados Unidos}

A pesar de ser una potencia en la estructura internacional, el comportamiento estadounidense en el ártico es totalmente defensivo. Strategic Outlook for the Artic, un documento guía de la armada estadounidense, coloca como objetivo principal la defensa de la influencia estratégica norteamericana en la región. En este sentido, la fuerza naval establece como prioridades ciertos aspectos, entre los cuales pueden mencionarse: defensa de la soberanía estadounidense, aseguramiento de una zona libre de conflicto, preservación de la libertad de navegación y promoción de la cooperación entre los países del área (The United States Navy, 2019) .

Durante la administración Obama, el presidente visitó Alaska en 2013 y se creó un comité especial para la coordinación de acciones estratégicas en el Ártico. Al igual que Canadá y Dinamarca, las estrategias nacionales árticas tratan sobre temas de seguridad, cooperación, desarrollo científico, cambio climático y pueblos indígenas (Department of Defense, 2013)

La presencia de las fuerzas armadas estadounidenses en el Ártico es mucho menor a la de Canadá, incluso si se trata de infraestructura militar, ya que los estadounidenses solo cuentan con dos rompehielos patrulla atracados en Seattle (Estrada, 2020, p. 21) . A pesar de la poca presencia, como se ha destacado anteriormente, Estados Unidos reconoce la importancia geopolítica del Ártico y las posibilidades de una carrera por convertirse en la potencia dominante. Sin embargo, descarta un conflicto directo. Aunque destaca el respeto por el cumplimiento del Derecho Internacional y el compromiso por garantizar un ambiente de paz de todos los Estados árticos, no duda en dejar claro expresamente estar preparados para responder ante cualquier riesgo de conflicto protegiendo su interés nacional (The United States Navy, 2019, p. 5) .

Estados Unidos no es partidario de establecer un Tratado Ártico en las mismas condiciones que concurren en el Tratado Antártico y afirma que no existen razones para considerar este tipo de régimen como necesario. Además, promociona la ratificación de la CONVE$M A R^{13}$ y el mantenimiento del Consejo Ártico como un foro de alto nivel (Suvanto, 2016) . Tiende, desde 2009, a promocionar la cooperación entre los Estados en la conservación ambiental y de los pueblos indígenas, lo que se explica por la posición que ostenta dentro de la estructura internacional y la pertenencia a foros internacionales como aliado de Canadá, Dinamarca y Noruega.

\section{Noruega}

Noruega, a través The Norwegian Government's High North Strategy aprobada en 2006, establece los objetivos referentes al Alto Norte y las estrategias para su cumplimiento, en la que se remarcan los aspectos siguientes: la cooperación cercana con Rusia, el combate a las actividades ilícitas en el área, desarrollo de los pueblos indígenas, recursos naturales y

13 Convención de las Naciones Unidas sobre el Derecho del Mar, en otros documentos aparece como UNCLOS por sus siglas en inglés. 
de pesca, a su vez plantea la necesidad de mostrar presencia y dominio en sus territorios insulares como Svalbard (Maritime Board, 2009). Es interesante que en el documentos se insiste en tres diferentes aspectos: el primero es la cooperación cercana con Rusia a través de una política exterior pragmática y cooperativa entre ambos, el segundo aspecto es el ejercicio de la soberanía y la autoridad a través de la presencia militar en la zona garantizando la seguridad marítima en términos integrales, y finalmente se insiste en convertirse en líderes de la región a través de la investigación científica, conservación del ambiente y cooperación.

A pesar de la buena fe y la cooperación bilateral con Rusia, en 2015 Noruega decide dejar la relación cercana con esta, en respuesta a la anexión de Crimea y el conflicto ucraniano (Heske, pág. 38). En 2020 el gobierno noruego publicó un nuevo libro blanco: The Norwegian Government's Arctic Policy. En este documento precisan los desafíos a la seguridad de su territorio ártico en la que destacan la situación de beligerancia rusa por medio de la ampliación de la infraestructura y armamento militar. Las maniobras militares de Moscú en la zona ártica han despertado, a su vez, actividades de disuasión de otros Estados aliados de la OTAN, como Estados Unidos, Reino Unido y Dinamarca, a través de la ampliación de los vuelos regulares sobre el Mar de Barents, cuestión que Noruega ve como contribución al equilibrio de poder frente a las acciones rusas (Ministry or Foreign Affairs, 2021) .

Así las cosas, Noruega ha sabido mostrar su buena voluntad para la solución de controversias y cooperación, sin descuidar sus objetivos geopolíticos en la región a través del ejercicio soberano en los territorios árticos a la vez que refuerza las relaciones con los Estados de la OTAN en la zona ártica. El Estado noruego reconoce que sus capacidades militares son limitadas, ${ }^{14}$ por lo que la alianza con otros Estados con un mejor posicionamiento dentro de la estructura internacional le es de beneficio. Ante la necesidad de una seguridad colectiva que mantenga el equilibrio en la región, su comportamiento defensivo y multilateralista puede generarle excelentes resultados en cuanto al ejercicio del liderazgo dentro del grupo ártico.

\section{Rusia}

Las pretensiones rusas sobre el Ártico datan desde los tiempos de la URSS. No obstante, en la actualidad estas pretensiones se establecen con énfasis en el posicionamiento de la ruta del Noreste como alternativa a Suez. Aunque no lo parezca, la ruta ha estado abierta al paso de naves desde 1987, pero las condiciones de las aguas congeladas no han permitido mayor paso en la zona, por lo que se volvió una ruta casi exclusiva de los rusos (Lojo Romero, 2018) .

14 Según el libro blanco el fortalecimiento militar de Noruega está basado en dos bandas principalmente, por un lado en la compra de nuevos aviones de combate F-35, aviones de patrulla P-8 y nuevos submarinos, por otro, los ejercicios y adestriamientos militares de sus tropas fuera de Noruega en el marco de los páises OTAN. 
Desde 2013 Rusia emprendió un constante posicionamiento y demostración de dominio en el Ártico mediante el despliegue y equipamiento de bases militares con misiles supersónicos en aquellos puntos que considera estratégicos. El gasto militar para armamento ruso se ha visto incrementado en ciertos años ${ }^{15}$, atendiendo a las iniciativas lanzadas por el gobierno con respecto a las estrategias en el Ártico (Bellaubí Pallarés, 2017) . Las pretensiones rusas sobre la región se pueden reducir a dos específicas: la jurisdicción sobre la Ruta del Noreste y la continuidad de su Zona Económica Exclusiva hasta las 350 millas, por lo que se ha extendido el refuerzo y la presencia de tropas en las islas de la Nueva Siberia (Estrada, 2020 , p. 10) , dando como resultado la molestia y alerta tanto de los países árticos como de la OTAN.

El Kremlin conoce la importancia estratégica de la zona y ante el deshielo polar considera que el territorio podría ser más vulnerable a amenazas extranjeras. En el programa de acción de 2013, Artic Strategy 2020, incluyó temas de cooperación, desarrollo de los pueblos indígenas y medidas contra en cambio climático. Sin embargo, en 2017 a raíz de la situación de sanciones y tensiones con algunos países europeos por el tema ucraniano, Rusia promulgó el Socioeconomic Development of the Russian Arctic Zone to 2025 con un contenido más securitario que incluye maniobras militares en la zona. En octubre de 2020 Moscú lanzó otro programa estratégico sobre el Ártico llamado Strategy for Developing the Russian Arctic Zone and Ensuring National Security through 2035, en el cual exponen las amenazas del cambio climático y deshielo ártico, incluyen la necesidad de mejorar las condiciones económicas de la población ruso-ártica a través de la explotación de recursos energéticos y a más largo plazo establecer la Ruta del Noreste como nueva ruta de transporte marítimo mundial (Kluge \& Paul, 2020) .

A diferencia de los programas anteriores, el plan hacia 2035 no incluye los aspectos que fueron expuestos en los documentos anteriores como la participación de las organizaciones civiles y aunque explora las posibilidades de cooperación con otros Estados, dedica mayor espacio a la seguridad y las amenazas entre las que destacan probables ataques a sus términales de petróleo y gas provenientes del Estrecho de Bering o desde los territorios daneses o noruegos. Por ello, reabrieron la mayoría de bases militares árticas de la URSS, cerradas en 1990.

\section{China}

Aunque China no es un país ártico como tal, ha logrado ser tomado en cuenta como miembro observador del Consejo del Ártico desde el 2013. Dadas las ventajas de tránsito marítimo y la cantidad de mercancías que el país oriental mueve de un continente a otro ${ }^{16}$,

15 Desde que Putin llegó al poder, el gasto militar ha tenido un "despegue" significativo, teniendo mayor inversión en el 2016 con la construcción de rompehielos. Para más información puede dirigirse a García Estrada, Ángel, Cambio Climático y Aproximación a las Potencias del Ártico, Artículo de Opinión Instituto Español de Estudios Estratégicos, figura 4, pág. 9

16 Se estima que el $80 \%$ de las mercancías a nivel mundial se transportan por vía marítima, considerándose el medio de transporte más barato. Lojo Romero, Raul, El Desarrollo del Transporte Marítimo en el Ártico, Trabajo de Grado en Naútica y Transporte Marítimo, Universidad de La Coruña, septiembre de 2018, pág. 49 
el acceso a una ruta con menos peligros y más corta es de gran importancia en el posicionamiento como potencia mundial. Conociendo sus capacidades económicas a nivel global, China invierte fuertemente en Islandia $y$, aunque no tiene acceso a territorios en el Polo Norte, es poseedor de un espacio geoestratégico importante a las puertas del océano Ártico y, por ende, de las rutas del norte (Fernández, 2018) .

En China's Artic Policy, un documento publicado por el gobierno chino sobre sus pretensiones en el Ártico, se sostiene la importancia de la región para el desarrollo de nuevas rutas marítimas, protección del medio ambiente y la investigación científica (State Council PRC, 2018) . El país oriental establece cinco líneas de acción con respecto a su participación en las relaciones árticas: exploración e investigación científica, protección del medio ambiente, respeto por la explotación de recursos en el marco de las leyes internacionales, gobernanza y cooperación internacional.

China es consciente de los efectos del deshielo polar. Las temperaturas provocadas por el calentamiento global afecta los patrones de precipitación chinos a raiz del desplazamiento de las corrientes frías hacia el sur de sus costas, modificando los patrones del monzón de verano (Rodrigo Calvo, 2019). En ese sentido, considera que los asuntos árticos no pueden ser tratados solamente por los páises interárticos, sino por los Estados fuera de la región y por la comunidad internacional a través de la sostenibilidad y beneficio mutuo. 


\section{Rutas marítimas árticas: la Ruta Noroeste y la Ruta Noreste}

\section{Mapa 3: Comparación ruta ártica vs. Suez}

\section{Nueva frontera \\ Cómo la Ruta Maritima del Norte se compara con la ruta tradicional via el canal de Suez}

\section{RUTA MARITIMA DEL NORTE}

Tiempo de travesia

35 dias

Peligros

Icebergs

Plazo para realizar el viaie

Entre julio y noviembre

Buques de carga de contenedores

Uno este aho

\section{RUTA DEL CANAL DE SUEZ}

Tiempo de travesia

48 dias

Peligros

El acceso al canal de Suez es riesgoso

debido a los disturbios en Egipto

Plazo para realizar el viaje

Todo elaño

Buques de carga de contenedores

17.000 el año pasado

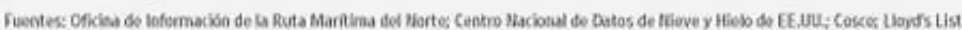

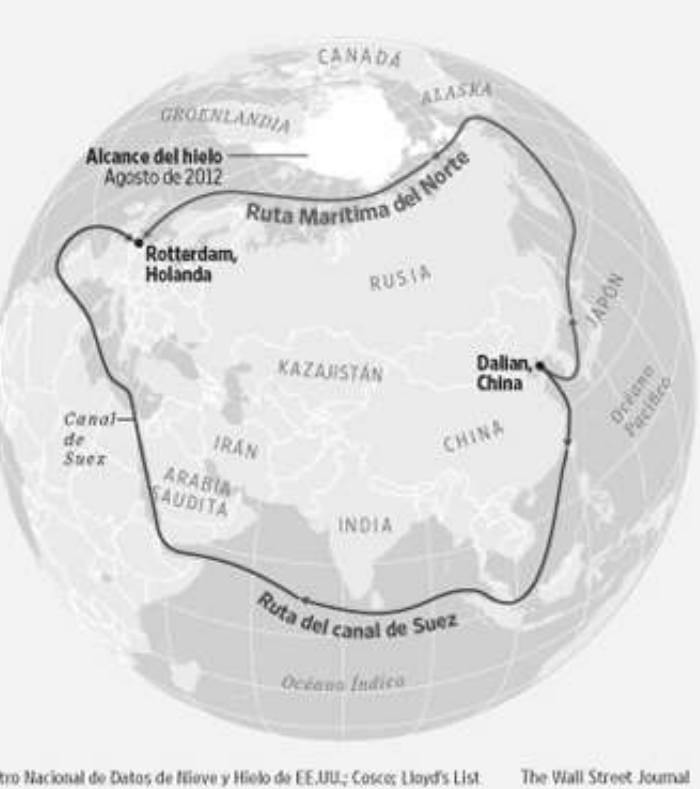

A diferencia de finales del siglo antepasado, cuando los estadounidenses compraron Alaska a los zares rusos, el Ártico nunca fue tan importante y estratégico en el posicionamiento del poder internacional como actualmente. Con el deshielo, las rutas del norte son una opción viable a mediano plazo para el transporte de mercancías, por lo que la jurisdicción sobre las aguas es un punto de ventaja para los Estados Árticos en la búsqueda de un mejor posicionamiento en la estructura internacional. A continuación, se describen cada una de las rutas que componen la Ruta del Norte.

\subsection{La Ruta Ártica y la Ruta de Suez}

El transporte marítimo actualmente presenta ciertos inconvenientes como el exceso de buques que transitan en una misma ruta, saturando choke points tan importantes como el Estrecho de Malaca, Ormuz, el Canal de Suez y el Canal de Panamá. Así, cualquier retraso en la logística del transporte de mercancías podría generar incontables pérdidas ${ }^{17}$. Igual 
riesgo se corre en aquellas rutas transitadas que sufren de la presencia de piratas, como el caso del Estrecho de Bab el Mandeb en las costas del Cuerno de África ${ }^{18}$.

El descubrimiento de nuevas rutas de tráfico más eficientes y seguras son un elemento clave en la economía y el comercio internacional. De esta manera, al reducirse la cantidad de hielo en el Océano Ártico, las masas de agua expuestas son una oportunidad importante para generar espacios de navegación más eficientes, como se presenta en el mapa 3, que compara la ruta "tradicional" que pasa por el Canal de Suez y el Mediterráneo, incluso Gibraltar si se desplaza hacia Occidente, con respecto a la ruta marítima del Noreste, es decir, pasando por los países árticos asiáticos y europeos.

A diferencia de las rutas ya establecidas, como la de Suez, Cabo de Buena Esperanza y Panamá, la ruta del Norte, sea a través del paso del noroeste por Canadá y Estados Unidos, o por el noreste atravesando los territorios siberianos en su mayoría, no es una ruta definida (Bellaubí Pallarés, 2017) . A pesar de que ofrece una posibilidad propicia en términos logísticos, presenta serias deficiencias que tienen relación con la época del año en que se puede navegar por estas aguas, aunque si el ritmo de deshielo se mantiene, se podría navegar por más tiempo (Zaragoza, 2014).

Si las rutas del norte estuviesen despejadas, los costos de navegación y transporte se acortarían hasta en un $50 \%$. Por ejemplo, si se compara la distancia de navegación de un contenedor desde Londres a Yokohama entre los diferentes choke points de mayor tráfico, el Canal de Panamá, Suez y Buena Esperanza ${ }^{19}$, con una distancia de 7.200 millas por la ruta marítima del norte entre Londres y Yokohama, en comparación con la ruta más distante, que sería el cabo de Buena Esperanza con 14.750 millas, la diferencia entre una y otra sería de un 205\% aproximadamente (Bellaubí Pallarés, 2017) .

en marzo de 2021. El canal de Suez es la ruta del 12\% del comercio mundial, por lo que esta situación retrasó la logística de muchas mercancías a nivel mundial. Egipto exige a la empresa japonesa Evergreen, dueña de la embarcación, una compensación calculada en \$1, 000 millones.

18 Según la Asociación de Navieros Españoles, en el 2017 se registró su punto más alto con 54 incidentes en comparación a los 27 del 2016. https://www.anave.es/prensa/ultimas-noticias/1805-las-cifras-de-pirateriaalcanzaron-en-2017-su-nivel-mas-bajo-en-22-anos-segun-el-ultimo-informe-anual-de-imb

19 Situados en América y África, por la ruta de África septentrional para Suez y África austral para el cabo de Buena Esperanza. 


\section{Mapa 3: Deshielo y las rutas marítimas árticas}

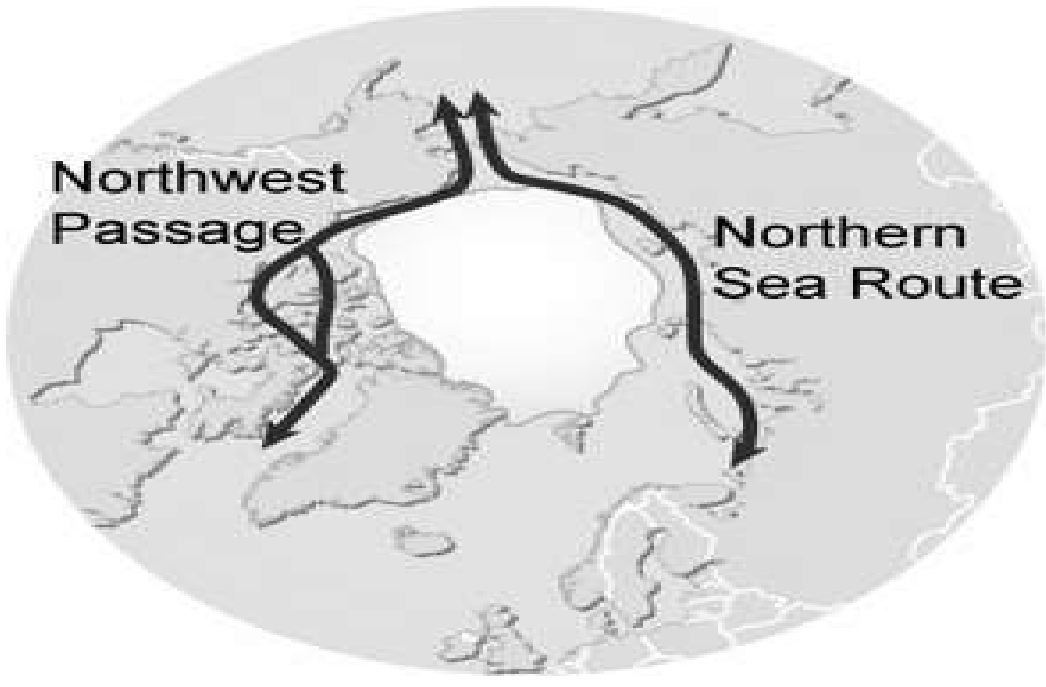

Fuente: Fundación Nuestro Mar https://www.nuestromar.org/antiguas/ruta-maritima-del-norte-se-abrioanticipadamente-en-el-artico/

Algunas empresas privadas de logística y transporte marítimo de contenedores iniciaron ensayos para transitar en esta ruta. La empresa danesa Maersk, por ejemplo, realizó viajes entre Vladivostok y San Petersburgo a través de portacontenedores rompehielos. Sin quedarse atrás y al igual que Maersk, la empresa china COSTCO ya hizo viajes por la ruta del Ártico. En 2012 el país asiático envió su primer navío bajo el cumplimiento de estándares sobre los buques navegables en hielo establecidos por la OMI y los parámetros propios de Rusia y China.

No obstante, en octubre de 2019 cinco navieras ${ }^{20}$ que transportan cerca del $64,5 \%$ de los contenedores a nivel mundial descartaron utilizar la ruta del Norte por dos razones principales: los altos costos de navegación por utilizar buques específicos y el impacto negativo al medio ambiente (Palau, 2019). Así las cosas, los Estados árticos tendrán que replantearse sus objetivos sobre las posibilidades de navegación para transporte de gran calado sobre la zona, como Rusia lo estableció en su programa estratégico descrito en el apartado anterior.

\subsection{Las Ruta del Norte: el paso de Noroeste y la Ruta del Noreste}

Separando las rutas árticas en dos rutas posibles conocidas, como la Ruta del Noroeste y la Ruta del Noreste (como se muestra en el mapa 4), se describen las características de cada una de ellas y las pretensiones de los Estados ribereños para su control y jurisdicción. 
La ruta del Noroeste tiene un tamaño aproximado de 3.200 millas náuticas que bordean las dieciocho mil islas del archipiélago ubicado en el hemisferio polar occidental. El paso entre los estrechos de las islas constituyen aguas interiores, por lo que la ruta estaría controlada por Canadá (Zaragoza, 2014, p. 238) . Los canadienses, a través de sus reclamaciones por la dorsal Lomonósov, pretenden ejercer soberanía sobre las aguas que comprenden la ruta. De ser así se posicionarían como un Estado fuerte a nivel económico y geopolítico, con mayores ventajas dentro de los organismos multilaterales como la OTAN, escalando en la estructura internacional como una potencia media importante. Esta pretensión no es compartida ni por Estados Unidos ni por la Unión Europea, quienes abogan por un libre tránsito en la región con régimen internacional, lo que exigiría la convergencia de los Estados interárticos y extrarregionales en esfuerzos y compromisos específicos para la conservación del ambiente y la seguridad marítima.

Por otra parte, la ruta del Noreste tiene una longitud de 2.500 millas náuticas y atreviesa varios de los mares del norte ${ }^{21}$, por lo que estaría controlada por dos Estados ribereños: Noruega y Rusia, el cual posee la mayor costa ártica (Zaragoza, 2014, p. 240). El Estado ruso tiene claros objetivos de posicionar la ruta como una alternativa a las rutas tradicionales. Sin embargo, con las últimas declaraciones de las cinco navieras más importantes tendrá que replantearse las posibilidades de ocurrencia en la escala popuesta.

\section{Perspectivas}

Las acciones de Canadá, Dinamarca, Noruega y Rusia contenidas en sus programas estratégicos muestran claridad en los objetivos y pretensiones sobre la región. Sin importar si el marco de acción versa sobre un comportamiento defensivo o no con respecto a su posición en la estructura internacional, conocen la importancia geopolítica en términos de recursos extractivos, biodiversidad y rutas marítimas. Los Estados Unidos, por su parte, muestra un comportamiento defensivo en el que promueve la cooperación regional para asegurar la libre navegación y la seguridad marítima. Sin embargo, hace sobrevuelos y patrullajes en el Mar de Barents frente a las maniobras militares rusas en la zona.

Los programas estratégicos más recientes de Noruega, Rusia y Canadá dejan en claro el aumento de las tensiones en la región. Estos tres Estados árticos refuerzan sus capacidades militares con la compra de armamento, presencia militar y entrenamiento a sus efectivos. En este sentido, el papel de la OTAN será determinante para mantener la disuasión y evitar incidentes entre las tropas de estos Estados.

En cuanto al cambio climático, la situación de contaminación marina podría verse exacerbada por el uso de plástico, que ha aumentado durante la pandemia de COVID19. Según Naciones Unidas, un 70\% del plástico utilizado termina como desecho en el mar. En 2020 con la pandemia se consumieron un promedio de 129.000 millones de mascarillas y 65.000 millones de guantes mensualmente en todo el mundo (Fusssteter, 2021). Con estas cifras 
es claro que los compromisos globales sobre medio ambiente y conservación de ecosistemas es totalmente necesaria, por lo que el Consejo Ártico tendrá que jugar un papel importante como foro de concertación de la región lo que estará condicionado a su vez por los intereses rusos al asumir la presidencia del Consejo en mayo del presente año (2021).

Así las cosas, el papel de las potencias medias en el Ártico sería esencial para lograr garantizar la seguridad marítima en la región reflejada en acuerdos que integren a todas las partes involucradas contando con la participación activa de organizaciones internacionales, organizaciones indígenas y socios estratégicos. El rol de China y su pretensión de mayor participación en los asuntos árticos podría hacerse realidad parcialmente: por una parte, la situación del cambio climático y la conservación de la fauna marina requerirá de compromisos fuertes más allá de los países interárticos en la conservación ambiental de la región, esfuerzos que a su vez deben de ser aprovechados con la reincorporación de Estados Unidos a las responsabilidades establecidas por el Acuerdo de París. No obstante, el país asiático estaría lejos de lograr un Tratado Ártico que permita un régimen internacional como lo propone.

Aunque las rutas árticas presentan un opción viable para los viajes marítimos transcontinentales, no se está ante una sustitución de la ruta de Suez o Panamá. El volumen de transporte en estas rutas podría disminuirse según los destinos a los que se transporte la mercancía; sin embargo, los choke points como Malaca, Ormuz, Suez y Panamá seguirán siendo representativos, ya que el transporte de mercancías y combustibles provenientes de otras zonas serán siempre significativas. Los costos de navegación y las consecuencias ambientales son altos, por lo que, al menos por hoy, aunque el tránsito por las rutas árticas sería económicamente viable hasta 2035 (British Antarctic Survey, 2020) , las principales navieras no atravesarían la zona. Las pretensiones de un posicionamiento mundial de las rutas del norte tendrían que ser replanteadas estableciendo objetivos a escala regional en el transporte de mercancías y en la búsqueda de inversiones sostenibles que podrían coadyuvar al desarrollo de los pueblos indígenas de la región.

En adelante, si el statu quo se mantiene en la zona, las estrategias de los Estados ribereños y sus pretensiones se seguirán actualizando; sin embargo, la necesidad de activar mecanismos que propicien las actividades sostenibles en el Ártico para aquellas empresas que cuentan con buques aptos para la navegación en las rutas del norte es por lo pronto indispensable, dado el estancamiento del comercio a raíz de la pandemia de COVID19, situación que ya ha dejado efectos recesivos preocupantes en la economía mundial.

\section{Conclusiones}

La vasta cantidad de recursos energéticos y biodiversidad del Ártico hacen de la zona un espacio de importancia geopolítica sobresaliente. Las pretensiones de los Estados ribereños y China son ajustadas a sus capacidades dentro de la estructura internacional. No obstante, en esta región se observa cómo las potencias medias ostentan un papel central en el equilibrio de poder de la zona. Aunque sus capacidades materiales (económicas y militares) no se comparan a las de Estados Unidos, Rusia y China optan por establecer políticas de posicionamiento y liderazgo en la zona a través de la cooperación en foros multilaterales como el Consejo del Ártico y otros acercamientos. 
La escalada de tensiones entre los países ribereños desde la situación de Ucrania y Rusia, ha demostrado que los Estados ribereños tuvieron que actualizar sus objetivos y estrategias en los programas planteados con anterioridad, estableciendo un marco de acción con énfasis en la seguridad y despliegue militar. Llama la atención la situación de Noruega y Rusia, con cambios drásticos en la política exterior de los noruegos hacia los rusos, la cual después de colocar la cooperación entre ambos como un pilar fundamental de su programa estratégico hoy opta por rescindir de tales lazos y, ante el despliegue ofensivo del Kremlin, fortalece la presencia militar a través de la renovación de armamentos y el adestriamiento de sus efectivos en el marco de la alianza OTAN.

Esta situación ralentiza los esfuerzos por combatir las consecuencias del cambio climático y la garantía de la seguridad marítima en la región, como se mencionó al inicio de este artículo. Muestra de ello es el programa estratégico ruso vigente, en el cual si bien existe un apartado sobre la cooperación internacional, se refiere a la atracción de inversión extranjera con miras a mejorar las condiciones de vida de la población rusa-ártica, pero no se enfoca en estrategias cooperativas con respecto a cambio climático y seguridad marítima.

No obstante, la voluntad de cooperar será clara cuando se asuma la presidencia del Consejo Ártico y este tema sea abordado. Aunque los países restantes son aliados, la poca anuencia de Rusia sería contraproducente, tomando en cuenta que el Estado posee la mayoría de costas árticas, sin olvidar el papel que juega en la estructura de poder internacional. La percepción de Rusia sobre las amenazas latentes a sus recursos ha generado una política exterior ofensiva a nivel global pero con mayor énfasis en las zonas que considera importantes como el Ártico. El escenario futuro requerirá de entendimientos entre los Estados ribereños y, así las cosas actualmente, países como Noruega y Dinamarca serán clave para que estos acercamientos y compromisos puedan hacerse realidad, en consecuencia, mejorarán su prestigio internacional como potencias medias esenciales en el mantenimiento de la paz en la región.

\section{Bibliografía}

Bellaubí Pallarés, I. (2017). Nuevas Rutas Árticas de Navegación Polar [Trabajo de fin de máster, Universidad de Cantabria]. https://repositorio.unican.es/xmlui/bitstream/handle/10902/12494/Bellaub\%C3\%AD\%20PaIlar\%C3\%A9s\%2C\%20Ingrid.pdf?sequence=1\&isAllowed=y

British Antarctic Survey. (2020, agosto 10). Past evidence supports complete loss of Arctic seaice by 2035. ScienceDaily. https://www.sciencedaily.com/releases/2020/08/200810113216.htm

Brzezinski, Z. (1998). El gran tablero mundial: La supremacía estadounidense y sus imperativos geoestratégicos (1a ed). Paidós.

Cuellar Laureano, R. (2012). Geopolítica. Origen del concepto y su evolución. Revista de Relaciones Internacionales, 113, 59-80.

Deparamento de Seguridad Nacional. (s. f.). Seguridad Marítima / DSN. Seguridad Nacional un proyecto compartido. Recuperado 21 de abril de 2021, de 
https://www.dsn.gob.es/es/sistema-seguridad-nacional/qu\%C3\%A9-es-seguridad-nacional/\%C3\%A1mbitos-seguridad-nacional/seguridadmar\%C3\%ADtima

Department of Defense. (2013). Artic Strategy. https://dod.defense.gov/Portals/1/Documents/pubs/2013_Arctic_Strategy.pdf

Estrada, G. (2020). Cambio climático y aproximación de las potencias al Ártico. 16, 27.

Fernández, E. (2018, abril 3). China se lanza a la conquista del Ártico. El Confidencial. https://www.elconfidencial.com/mundo/2018-04-03/china-se-lanza-a-la-conquista-del-artico_1543864/

Fusssteter, F. (2021, marzo 30). El uso exagerado del plástico durante la pandemia de COVID19 afecta a los más vulnerables. Noticias ONU. https://news.un.org/es/story/2021/03/1490302

Geoenciclopedia. (s. f.). Océano Ártico-Información y Características. Recuperado 28 de abril de 2021, de https://www.geoenciclopedia.com/oceano-artico/

Godio, L. (2019). El derecho del mar y el cambio climático en el Ártico: ¿estrechos internacionales o protección y preservación del medio marino? Anuario Colombiano de Derecho Internacional, 12, 19-42. https://doi.org/10.12804/revistas.urosario.edu.co/acdi/a.7567

Gómez, I. F. (2019). (Re) militarización del Ártico: ¿Cautivos de un dilema de seguridad? [Trabajo fin de grado, Universidad de Barcelona]. https://www.recercat.cat/bitstream/handle/2072/362319/Fern\%C3\%A1ndez\%20G\%C3\%B3mez \%2C\%20lv\%C3\%A1n.pdf?sequence=1

Government of Canada. (2019, junio 14). Canada's Arctic and Northern Policy Framework [Policy]. https://www.rcaanc-cirnac.gc.ca/eng/1560523306861/1560523330587

Government of Kingdom of Denmark. (2011). Kingdom of Denmark Strategy for the Arctic 2011-2020. https://um.dk/ /media/um/english-site/documents/politics-anddiplomacy/greenland-and-the-faroe-islands/arctic\%20strategy.pdf?la=en

Greenpeace. (2014). Ártico, el océano más desprotegido del planeta. http://archivo-es.greenpeace.org/espana/Global/espana/2015/Report/artico/artico-el-oceano-masdesprotegido-BR.pdf

Hernández, S. (2008). LA TEORIA DEL REALISMO ESTRUCTURALISTA Y LAS INTERACCIONES ENTRE LOS ESTADOS EN EL ESCENARIO INTERNACIONAL. Revista Venezolana de Análisis de Coyuntura, 12, 13-29.

Iturralde, M. F. N. (2019). La cuestión Ártica. Revista AFESE, 65 (65), Article 65. http://www.revistaafese.org/ojsAfese/index.php/afese/article/view/1174

Jørgensen-Dahl, A. (2010). ARCTIS / Petróleo y gas del Ártico. Arctics Knowledge Hub. http://www.arctis-search.com/Arctic+Oil+and+Gas

Kluge, J., \& Paul, M. (2020, noviembre). Russia's Arctic Strategy through 2035. 
https://www.swp-berlin.org/10.18449/2020C57/

Larrucea, J. R. de. (2015). Seguridad marítima: Teoría general del riesgo. MARGE BOOKS. https://books.google.com.sv/books?hl=es\&lr=\&id=jH89CgAAQBAJ\&oi=fnd\&pg =PA11\&dq=seguridad+mar\%C3\%ADtima\&ots=YV5WA-cyz0\&sig=xiebypcKnMjARTyvnt5Y3qvpJ|4\&redir_esc=y\#v=onepage \&q=seguridad\%20mar\%C3\%ADtima\&f=false

Lojo Romero, R. (2018). El desarrollo del transporte marítimo en el Ártico. https://ruc.udc.es/dspace/handle/2183/21197

Londoño, J. (2018). Fundamentos de la Geopolítica. Barnes \& Noble Press.

Maritime Board. (2009). Strategic Action Program. Ministry for Economic Development, 26.

Ministry or Foreign Affairs. (2021, enero 26). The Norwegian Government's Arctic Policy [Rapport]. Government.No; regjeringen.no. https://www.regjeringen.no/en/dokumenter/arctic_policy/id2830120/

National Snow and Ice Data Center. (s. f.). Quick Facts on Arctic Sea Ice / National Snow and Ice Data Center. Quick Facts. Recuperado 20 de abril de 2021, de https://nsidc.org/cryosphere/quickfacts/seaice.html

Palacian de Inza, B. (2010, octubre). La creciente importancia del Ártico. Revista Española de Defensa, 50-53.

Palau, J. C. (2019, octubre 23). Las principales navieras de contenedores descartan por ahora usar la ruta del Ártico. El Mercantil. https://elmercantil.com/2019/10/23/lasprincipales-navieras-de-contenedores-descartan-por-ahora-usar-la-ruta-delartico/

Rodríguez, H. (2018, diciembre 7). El deshielo del Ártico se acelera sin precedentes. www.nationalgeographic.com.es. https://www.nationalgeographic.com.es/ciencia/actualidad/deshielo-artico-se-acelera-sin-precedentes_13620

Sánchez, J. (2021, marzo 16). Animales en peligro de extinción del Polo Norte-LISTA y VíDEOS. ecologiaverde.com. https://www.ecologiaverde.com/animales-en-peligro-de-extincion-del-polo-norte-1604.html

State Council PRC. (2018, enero 26). Full text: China's Artic Policy. State Council The People Republic of China. http://english.www.gov.cn/archive/white_paper/2018/01/26/content_281476026660336.htm

Suárez Llanos-Galán, F. (2009). La Seguridad Marítima en la Marina Civil. En Instituto Español de Estudios Estratégicos (Ed.), Impacto de los riesgos emergentes en la seguridad marítima. Ministerio de Defensa, Secretaría General Técnica.

Suvanto, V. P. (2016). Geopolitics of the Arctic: Challenges and Prospects. 62.

The United States Navy. (2019). Navy Strategic Outlook Artic. https://media.defense.gov/2020/May/18/2002302034/-1/-1/1/NAVY_STRATEGIC_OUTLOOK_ARCTIC_JAN2019.PDF

USCG. (2008). Fact Sheet (Fact Sheet N. ${ }^{\circ}$ 3049; Fact Sheet). Servicio Geológico de los Estados 
Unidos. https://pubs.usgs.gov/fs/2008/3049/fs2008-3049.pdf

Valencia, A. R., \& Ruvalcaba, D. E. M. (2010). Potencias medias y potencias regionales en el Sistema Político Internacional: Dos modelos teóricos. 1, 29.

Zaragoza, G. S. (2014). Las Nuevas Rutas Comerciales A Través Del Ártico. Una Consecuencia Del Cambio Climático. Revista General de la Marina, 266 (2), 233-249. 